\title{
CRECIMIENTO POSTRAUMÁTICO EN PACIENTES ONCOLÓGICOS
}

\author{
Gema Costa-Requena \\ Francisco Luis Gil Moncayo
}

Unidad de Psico-Oncologla. Hospital Durán I Reynals

\section{RESUMEN}

El objetivo de este estudio es aveniguar las propiedades psicométricas de la adaptación española del Inventario de Crecimiento Postraumático (PTGI) en una muestra de pacientes oncológicos ambulatorios $(N=130)$. El cuestionario permite valorar los cambios positivos que pueden percibirse tras un acontecimiento traumático como es el diagnostico o tratamiento de una enfermedad oncológica. Aplicamos un análisis factorial de componentes principales seguido de un análisis con el modelo de ecuaciones estructurales para contrastar la estructura factorial. Realizamos una comparación de medias y análisis correlacional entre las puntuaciones del PTGI y caracteristicas sociodemográficas o médicas.

Del análisis de componentes principales extraemos 4 factores que explican el $71 \%$ de la varianza total, del modelo de ecuaciones estructurales se corrobora la estructura monofactorial del cuestionario $\left(X^{2}=57,53 ; p=0,06\right)$ que da cuenta del $54,47 \%$ de la varianza. No aparecen diferencias significativas al comparar crecimiento postraumático con género, tratamiento médico recibido $y$ estadio de la enfermedad. Tampoco las

\footnotetext{
Correspondencia: Unidad de Psico-Oncologia. Hospital Duran I Reynals. Avd. Gran Via s/n, km 2,7. 08907 L'Hospitalet. Barcelona. Teléfono: 933357 011-ext. 3717. Fax. 932607783. Correo electrónico: gcosta@ico.scs.es.

Este estudio forma parte del proyecto que lleva por título Adaptación Psicologica y Morbilidad Psiquiátrica en Pacientes con Cáncer financiado por la Agéncia d'Avaluació de Tecnologla i Recerca Médiques (No Proyecto: 102/19/2004).
} 
correlaciones son significativas entre el PTGI y edad, tiempo desde el diagnóstico o malestar emocional.El inventario de crecimiento postraumático permite evaluar la percepción de cambios positivos tras el diagnóstico o tratamiento de una enfermedad oncológica, aunque en la muestra de este estudio el cambio no se define según la dimensionalidad propuesta por los autores del cuestionario.

Palabras clave: CRECIMIENTO POSTRAUMATICO; CARACTERISTICAS PSICOMÉTRICAS; CANCER; BENEFICIOS PERCIBIDOS.

\section{SUMMARY}

The main aim of this study was to assess the psychometric properties of the Spanish Posttraumatic Growth Inventory (PTGI) in a sample of cancer outpatients $(N=130)$. The questionnaire attempts to evaluate the perceived positive changes following the diagnosis or treatment of cancer patients. We have used the factor analysis of principal components, and after it, an analysis with the structural equations model to contrast the factorial structure. Also, we did a descriptive and correlation analysis between the PTGI scores and then demographic and medical variables. Through the component principal analysis, we found 4 factors, that explain $71 \%$ of the total variance. With the confirmatory factor analysis, we have confirmed the monofactorial structure of the questionnaire $\left(X^{2}=57,53 ; p=0,06\right)$, that explain $54,47 \%$ of the total variance. We did not find any statistical difference in posttraumatic growth comparing gender, medical treatment and medical stage. Neither, we found any significant correlation between PTGI and age, time since the diagnosis or distress.

The PTGI is a validated questionnaire to assess the perception of positive changes after the cancer diagnosis and treatment. However, in our study we found a difference dimensionality of the PTGl questionnaire, compared with the original one.

Key words: POSTTRAUMATIC GROWTH; PSYCHOMETRIC PROPIERTIES; CANCER; BENEFICIT PERCENED. 


\section{INTRODUCCIÓN}

El diagnóstico o tratamiento del cáncer puede ser entendido como un acontecimiento traumático, en el sentido que representa una amenaza a la integridad física de la persona o implica un peligro a la propia vida (APA, 1995). Mientras algunas personas ante situaciones vitales traumáticas llegan a desarrollar trastornos emocionales, otras son capaces de fortalecer sus recursos personales y adaptarse a las adversidades e incluso cambiar hacia la madurez o crecimiento personal (Cordova y cols., 2001). El beneficio percibido tras el acontecimiento traumático no está relacionado con la respuesta adaptativa entendida como índice global de calidad de vida o salud física percibida (Helgeson y cols., 2006).

Tedeschi y Calhoum (1995) definen crecimiento postraumático como la percepción de los cambios positivos que resultan del esfuerzo personal tras una crisis vital o acontecimiento traumático. Este cambio supone una transformación personal en la manera de responder ante las demandas vitales que conlleva un bienestar no apreciado antes de ocurrir el suceso traumático. El modelo cognitivo propuesto por estos autores, basado en las teorlas de afrontamiento del estrés, perrnite identificar los mecanismos y factores desencadenantes del crecimiento postraumático (Calhoum y Tedeschi, 2006), desde este modelo confeccionaron un instrumento para evaluar crecimiento postraumático, PostTraumatic Growth Inventory (PTGl; Tedeschi y Calhoun, 1996).

Ante un evento traumático que supone una amenaza de muerte o daño a la integridad física de uno mismo o los demás, una persona puede reaccionar con sentimientos de miedo y desesperanza, que conllevan un cambio en las asunciones básicas sobre sí mismo y el mundo. El acontecimiento traumático conileva una crisis existencial que desencadena un proceso de urumiación constructiva» con una búsqueda de significado dentro de una gran incertidumbre y vulnerabilidad personal. El proceso cognitivo o rumiación constructiva puede suponer con el tiempo cambios positivos en la visión de uno mismo, el sentido de relación con los demás y la filosofia ante la vida.

La percepción de cambios positivos tras un evento traumático no depende del mero paso del tiempo, sino que se ha relacionado con 
caracteristicas sociodemográficas de la persona y caracteristicas del evento traumático. Respecto al género algunos estudios con muestras de pacientes oncológicos no encuentran diferencias entre hombres y mujeres en crecimiento postraumatico (Ho y cols., 2004), en cambio en otros estudios las mujeres suelen experimentar más percepción de cambio positivo que los hombres tras el diagnostico o tratamiento de la enfermedad oncológica (Calhoun y Tedeschi, 2006; Thornton y cols., 2006; Jaarsma y cols., 2006). Con la edad, los resultados tampoco son aclaratorios, hay estudios que no encuentran relación entre la edad y puntuaciones en crecimiento postraumático (Tedeschi y Calhoun, 1996; Thornton y cols., 2006), en otros estudios son las personas más jóvenes las que experimentan mayor crecimiento postraumático (Jaarsma y cols., 2006; Bellizi y cols., 2006; Manne y cols., 2004). No parece haber relación entre tiempo desde que aconteció el evento traumático y crecimiento postraumático (Tedeschi y Calhoun, 1996) o las correlaciones son negativas (Weiss, 2004). Tampoco resulta clarificadora la relación entre malestar emocional y crecimiento postraumático, puntuaciones altas en el PTGI no necesariamente se acompañan de menos sintomatología ansiosa 0 depresiva (Jaarsma y cols., 2006), sin embargo otros datos parecen sugerir que la presencia de crecimiento postraumático es un indicador de que la persona tiene una vida más plena y significativa (Calhoun y Tedeschi, 2006; Ho et al., 2004).

La intensidad o severidad del acontecimiento traumático correlaciona más con cambios positivos tras su superación, al suponer que conlleva más actividad cognitiva (Calhoun y Tedeschi, 2006; Tedeschi y Calhoun, 1996), teriendo en cuenta además que el coritenido de las cogniciones se relacionan con una búsqueda de significado o reconfiguración de objetivos vitales (Bellizi y cols., 2006). En este sentido, son las estrategias de afrontamiento positivas y activas las que fomentan mayor crecimiento postraumático (Thornton y cols., 2006; Ho et al., 2004; Bellizi y cols., 2006). Teniendo en cuenta estos datos los objetivos de este estudio son: a) contrastar empiricamente la estructura dimensional del PTGI; b) determinar la relación entre malestar emocional y crecimiento postraumático, suponemos una correlación negativa; c) estudiar la relación entre variables sociodemográficas (género, edad y tiempo desde el diagnóstico) y 
variables médicas (tratamiento oncológico recibido y estadio de la enfermedad) con las dimensiones del inventario de crecimiento postraumático. Siguiendo los resultados propuestos por los autores del cuestionario (Tedeschi y Calhoun, 1996), planteamos que las mujeres informarán de más beneficios tras el diagnóstico o tratamiento de la enfermedad oncológica que los hombres. Ni la edad, ni el tiempo desde que ocurrió el evento traumático correlacionan con puntuaciones significativas en el PTGI. Asimismo, se espera que cuanto más severo es el acontecimiento traumático más crecimiento postraumático experimentará la persona, como medida de gravedad del diagnóstico utilizamos el estadio de la enfermedad (extensión local, loco-regional o metastático del tumor), mientras la severidad del tratamiento es evaluada por el tipo de tratamiento recibido (quimioterapia o radioterapia).

\section{SUJETOS Y MÉTODO}

\section{Sujetos/Muestra}

Este estudio se realizó en el hospital Duran y Reynals (Barcelona), perteneciente al Instituto Catalán de Oncología, durante los meses de abril del 2006 a enero del 2007. Se extrae aleatoriamente una muestra de pacientes oncológicos ambulatorios, que acuden a hospital de dia para recibir tratamiento de quimioterapia, o son visitados en las unidades funcionales de consultas externas. Tras explicarles los objetivos del estudio, se les ofreció participar en él, los pacientes que accedieron firmaron la hoja de consentimiento informado. El estudio fue aprobado por el comité de ética del hospital. Los criterios de inclusión fueron, pacientes ambulatorios con diagnóstico de enfermedad oncológica, y con una edad comprendida entre los 18 a 80 años. Asimismo, los criterios de exclusión fueron presencia de trastorno psicótico, una capacidad funcional menor del $40 \%$, según Karnofsky Performance Status (Karnofsky y cols., 1948) y deterioro cognitivo, con más de dos errores en el Test Pfeiffer (Pfeiffer, 1975). El tamaño muestral es calculado según criterios minimos para aplicar un análisis factorial, al menos cinco sujetos por ítem (Gorsuch, 1983). 
Se realiza una entrevista de evaluación donde se recogieron datos sociodemográficos del paciente (edad, sexo, estado civil, profesión, nivel educativo), datos médicos (antecedentes psiquiátricos del paciente, antecedentes oncológicos familiares, éxitus familiares por la enfermedad oncológica, diagnóstico clínico actual, estadio y tratamiento oncológico recibido) y se evaluó la percepción de gravedad que el paciente tenía sobre su enfermedad con una escala análogo visual puntuada de 1 (totalmente conocedor) a 4 (no conocedor), con ello se intenta recoger el grado de información que manifiesta el paciente sobre su enfermedad oncológica. Los datos sociodemográficos y médicos son contrastados con la historia clínica de cada paciente. Después de la entrevista se administran la escala de Ansiedad y Depresión Hospitalaria (HADS, Zigmoth y Snaith, 1983) y el PTGI, para que el paciente los cumplimente.

\section{Material}

El Inventario de Crecimiento postraumático (PTGI, Posttraumatic Growth Inventory; Calhoun y Tedeschi, 2006; Tedeschi y Calhoun, 1996), es un cuestionario de 21 items que evalúa la percepción de beneficios personales en supervivientes de un acontecimiento traumático. El formato de respuesta es de tipo likert con seis categorías, en un rango de puntuación de 0 (no cambio) a 5 (muy alto grado de cambio) en sentido positivo, a más puntuación mayor cambio percibido. Está constituido por cinco dimensiones (Tedeschi y Calhoum, 1996): Factor 1, denominado Relación con los demás (17\% de varianza total, $\alpha=0,85$ ), formado por los Items $6,8,9,15,16,20$ y 21: este factor evalúa el hecho de que la persona se sienta más unida y cercana a los demás ante las dificultades, incremente el sentido de compasión hacia los que sufren, y haya una voluntad para dejarse ayudar y utilizar el apoyo social que antes habla ignorado. Factor 2 , denominado Nuevas Posibilidades ( $16 \%$ de varianza total, $\alpha=0,84$ ) formado por los ítems 3, 7, 11, 14 y 17; referido a un cambio en los intereses $o$ actividades, algunas personas incluso emprenden nuevos proyectos vitales (ej, voluntariado). Factor 3, Fuerza Personal (11\% de la varianza total, $\alpha=0,72$ ) formado por los ítems $4,10,12,19$; este factor evalúa en qué medida la persona siente que ha sobrevivido a lo 
peor, confirmando con ello su fortaleza, se siente segura de sí misma y capacitada para superar dificultades, se resume con la expresión soy más vulnerable de lo que pensaba, pero más fuerte de lo que imaginaba. Factor 4 , denominado Cambio Espiritual ( $9 \%$ de la varianza total, $\alpha=0,85$ ) formado por los ítems 5 y 18; este factor agrupa la búsqueda de sentido espiritual de las cosas, búsqueda de significado a la vida y planteamientos existenciales de la persona. Finalmente el factor 5 Apreciación por la Vida ( $9 \%$ de la varianza total, $\alpha=0,67$ ), formado por los ítems 1,2 y 13; supone una actitud más relajada ante la vida, cambio en el estilo de vida (ej, hábitos saludables) o cambio en las prioridades vitales (ej, valorar pequeñas cosas). La adaptación española del cuestionario fue validada en población latina inmigrante (Weiss y cols., 2005). La solución factorial mostró una agrupación de tres factores diferente a los cinco propuestos por los creadores del inventanio. Factor 1, denominado Filosofía ante la Vida (explica el 23,9\% de la varianza total, $\alpha=0,85$ ) formado por los items $18,19,13,16$ y 1 . El factor 2, denominado SI mismo/Actitud positiva ante la vida $(23,4 \%$ de la varianza, $\alpha=0,87$ ) agrupa los ítems $7,3,4,11,14$ y 10 . El tercer factor Relaciones Interpersonales $(19,4 \%$ de la varianza, $\alpha=0,80)$ formado por los ítems 9 y 6 .

La Escala de Ansiedad y Depresión Hospitalaria (Zigmoth y Snaith, 1983), es un autoinforme de 14 items que evalúa sintomatologia de ansiedad y depresión con dos subescalas de 7 ítems cada una, puntuadas en una categoría de respuesta de 0 (nada) a 3 (mucho) según intensidad de la sintomatologia. Fue diseñada para pacientes con enfermedades médicas, no psiquiátricas. Por su brevedad y fácil comprensión es utilizada reiteradamente en psico-oncología para evaluar malestar emocional. La versión española del HADS (LópezRoig y cols., 2000) ha sido validada en población oncológica obteniendo buenos parámetros de fiabilidad y validez $(=0,85$ para la subescala de ansiedad y $\alpha=0,87$ para depresión).

\section{Análisis estadístico}

Se trata de un estudio no-experimental con método selectivo y diseño ex post facto retrospectivo simple. En el análisis estadístico, primero realizamos una descripción de los ítems, se presenta la 
distribución de frecuencias, media, mediana y desviación típica de cada item. Son considerados items adecuados aquellos con una desviación típica superior a 1 y una media situada alrededor del punto medio de la escala. Calculamos el índice de discriminación corregido con el coeficiente de correlación entre la puntuación en el item y el total de la escala.

Para estudiar la dimensionalidad del cuestionario utilizamos un análisis de componentes principales con rotación ortogonal varimax. Se valoró la bondad de ajuste de los datos analizados a un modelo factorial con la medida de adecuación muestral Kaiser-Meyer-Olkin (KMO) y la prueba de esfericidad de Barlett. Tras el análisis factorial se mantienen los valores propios mayores a uno, según criterio Kaiser. En la solución del análisis factorial definimos los siguientes criterios: tomar como significativos en cada factor saturaciones de los ítems a partir de 0,40 ; obtener el menor número de ítems sin saturar en factor alguno y el mínimo número de solapamientos. Para contrastar el modelo extraído del análisis factorial exploratorio se utilizó el modelo de ecuaciones estructurales (Ramos y cols., 2004), la estimación de los parámetros del modelo se realizó con el procedimiento de máxima verosimilitud. En este caso las medidas del ajuste absoluto determinan el grado en que el modelo conjunto predice la matriz de correlación o covarianza observada. Entre las medidas de ajuste utilizadas en el modelo de ecuaciones estructurales la fundamental es el estadístico ratio de verosimilitud chi-cuadrado $\left(X^{2}\right)$, con este estadístico se buscan diferencias no significativas entre las matrices actuales y las previstas lo que indicaria que el modelo propuesto se ajusta bien, o tiene la capacidad de predecir la matriz de varianzas y covarianzas entre las variables superficiales (items). Otra medida es el índice de bondad del ajuste (GFI), representa la cantidad de variabilidad que es explicada por el modelo. El índice ajustado de bondad del ajuste (AGFI) es un valor que tiene en cuenta los grados de libertad del modelo propuesto y el número de variables superficiales. Finalmente, el error de aproximación cuadrático medio (RMSEA), es representativo de la bondad de ajuste que podría esperarse si el modelo fuera estimado con la población no solo con la muestra extraida. Se midió la consistencia interna del PTGI con el coeficiente alfa de Cronbach (Cronbach, 1951) de los ítems en cada dimensión del cuestionario. 
Al estudiar la relación entre crecimiento postraumático y variables sociodemográficas calculamos el coeficiente de correlación de Pearson entre las dimensiones del PTGI y tiempo desde el diagnóstico, y edad. Además con el coeficiente de correlación intentamos averiguar la relación entre crecimiento postraumático y malestar emocional, evaluado con el HADS. Suponemos que una puntuación mayor en las dimensiones de crecimiento postraumático supondrá menor malestar emocional.

Como medidas de intensidad del acontecimiento traumático ante el diagnóstico de la enfermedad oncológica valoramos su estadio, para evaluar la severidad del tratamiento médico se considera si ha realizado o no, quimioterapia o radioterapia.

En el estudio de la relación entre crecimiento postraumático, género y tratamiento médico actual (quimioterapia y radioterapia), utilizamos una comparación de medias con la prueba $\mathrm{T}$ de student, previamente comprobamos la igualdad de varianzas con la prueba de Levene. Al comparar crecimiento postraumático y estadio de la enfermedad calculamos el análisis de varianza de un factor al ser el estadio una variable independiente con más de dos categorías de respuesta (en este caso se diferencia el estadio local, loco-regional, metastático y tumor no sólido).

Los datos fueron analizados con el programa estadístico SSPS versión 11 y con el paquete estadístico complementario AMOS 6.0. En los casos con valores perdidos, se elige la opción por defecto del programa estadistico, los ítems con ausencia de datos fueron excluidos del análisis, sólo se incluyen los casos con puntuación válida en todas las variables seleccionadas.

\section{RESULTADOS}

La muestra está formada por 130 pacientes que accedieron a participar en el estudio y auto-cumplimentar los cuestionarios, de ellos el $43 \%$ son hombres y el $57 \%$ son mujeres, con una media de edad de 47 años $(S x=10,5)$, otras características sociodemográficas y médicas se presentan en la tabla 1. Los cuestionarios fueron bien aceptados y auto-cumplimentados por los sujetos de la muestra.

El análisis descriptivo de los items se presentan en la tabla 2, en la discriminación de cada ítem con el total se obtienen valores adecuados que oscilan entre 0,53 y 0,81 . 
Tabla 1. Datos sociodemográficos $(N=120)$

\begin{tabular}{|c|c|c|}
\hline & $\begin{array}{c}\mathrm{N}^{\circ} \\
\text { Pacient }\end{array}$ & $\%$ \\
\hline \multicolumn{3}{|l|}{ Edad } \\
\hline Media & \multirow{3}{*}{\multicolumn{2}{|c|}{$\begin{array}{c}47,5 \\
10,5 \\
48\end{array}$}} \\
\hline Desv.Tip. & & \\
\hline Mediana & & \\
\hline Rango & \multicolumn{2}{|c|}{$23-73$} \\
\hline \multicolumn{3}{|l|}{ Género } \\
\hline Varón & 56 & 43,1 \\
\hline Mujer & 74 & 56,9 \\
\hline \multicolumn{3}{|l|}{ Educación } \\
\hline Estudios Primarios & $\begin{array}{l}45 \\
50\end{array}$ & 38,5 \\
\hline Educación Secundaria & 20 & 38,3 \\
\hline Formación Especializada & $\begin{array}{l}20 \\
15\end{array}$ & $\begin{array}{l}10,4 \\
11,5\end{array}$ \\
\hline Estudios Universitarios & & \\
\hline \multicolumn{3}{|l|}{ Estado civil } \\
\hline Casado/con Pareja & 20 & 15,4 \\
\hline Soltero & 3 & 2,3 \\
\hline Viudo & 8 & 62 \\
\hline Separado/Divorciado & 8 & \\
\hline $\begin{array}{l}\text { Antecedentes Psiquiduricos } \\
\mathrm{Si}\end{array}$ & 40 & 30,8 \\
\hline \multicolumn{3}{|l|}{ Antecedentes oncológicos familiares } \\
\hline $\mathrm{Si}$ & 78 & 60 \\
\hline Éxitus familiares por cáncer & 61 & 46,9 \\
\hline \multicolumn{3}{|l|}{ Estadio Actual } \\
\hline Local & 29 & 22,3 \\
\hline Loco-Regional & 43 & 33,1 \\
\hline Mestastásico & 36 & 27,7 \\
\hline No-Sólido & 22 & 16,9 \\
\hline \multicolumn{3}{|l|}{ Localización del Tumor } \\
\hline Órganos respiratorios e Intratorácicos & 16 & 12,3 \\
\hline Mama & 37 & 28,5 \\
\hline Genitourinarios & 20 & 15,4 \\
\hline Tejido limfático y Hematológico & 22 & 16,9 \\
\hline Órganos Digestivos y Peritoneo & 28 & 21,5 \\
\hline Labio, Oral y Faringe & 4 & 3,1 \\
\hline Otros & 3 & 2,3 \\
\hline \multicolumn{3}{|l|}{ Tratamiento Previo } \\
\hline Cirugia & 79 & 60,8 \\
\hline Radioterapia & 42 & 32,3 \\
\hline Quimioterapia & 49 & 37,7 \\
\hline Braquiterapia & 2 & 1,5 \\
\hline
\end{tabular}




\begin{tabular}{|lcc|}
\hline Tratamiento Actual & & \\
Radioterapia & 14 & 10,8 \\
Quimioterapia & 110 & 84,6 \\
\hline Introspección de la Enfermedad & 129 & 99,2 \\
$\quad$ Totalmente & 1 & 0,8 \\
$\quad$ Parcialmente & & \\
\hline Indice Karnousky (0-100) & 1 & 0,8 \\
50-Requiere ayuda de otros & 5 & 3,8 \\
60-Necesita ayuda ocasional de otros & 28 & 21,5 \\
70-Cuida de si mismo. Incapaz de actividad normal & 61 & 46,9 \\
80-Actividad normal con esfuerzo & 35 & 26,9 \\
90-Actividad normal. Signos leves de enfermedad & & \\
\hline Tiempo desde el diagnóstico & 31 & 24,2 \\
Menos de 3 meses & 27 & 21,1 \\
De 3 a 6 meses & 21 & 16,4 \\
De 6 a 12 meses & 49 & 38,3 \\
Más de 12 meses
\end{tabular}

El valor de la medida de adecuación muestral se considera bueno, con un índice KMO de 0,91; la prueba de esfericidad de Barlett obtiene un valor $X^{2}=1869,8(p<0,000)$ estadísticamente significativa, por lo que resulta pertinente utilizar el análisis factorial con estos datos. Aplicamos el análisis factorial de componentes principales con rotación ortogonal (varimax), con ello se extrajeron 4 factores con valores propios mayores a uno que explican el $71,61 \%$ de la varianza total, véase tabla 3. Estos factores explican cada uno el $54,47 \%, 6,40 \%$, $5,88 \%$ y $4,85 \%$ de la varianza respectivamente.

Dados estos resultados nos planteamos la posibilidad de hipotetizar sobre una estructura monofactorial del cuestionario. Siguiendo el principio de parsimonia del análisis factorial, al seleccionar el número de factores necesarios para captar una cantidad razonable de información, vemos que con sólo el primer factor se explica más del $54 \%$ de la varianza total, además ítems con carga factorial significativa en otros factores tienen saturaciones mayores de 0,4 en el primer factor (ítem 9, 13, 8, 6, 16), véase tabla 3 . El porcentaje de varianza oniginal explicada por el primer factor, nos indicó la necesidad de probar la estructura monofactorial del cuestionario con el modelo de ecuaciones estructurales en los 12 ítems con saturaciones significativas dentro del primer factor. El coeficiente de fiabilidad para la estructura monofactorial es $\alpha=0,952$. Los indicadores de ajuste 
Tabla 2. Valores del coeficiente de correlación item-escala, mediana, media y desviación típica de los items

\begin{tabular}{|l|l|c|c|c|c|c|}
\hline \multicolumn{2}{|l|}{ Iterns } & N & \multicolumn{2}{l|}{$\begin{array}{l}\text { Mediana } \\
\text { DSx }\end{array}$} & Media \\
\hline 1. & $\begin{array}{l}\text { Cambié mis prioridades sobre lo que es } \\
\text { importante en la vida }\end{array}$ & 129 & 3 & 2,81 & 1,58 &, 60 \\
\hline 2. & $\begin{array}{l}\text { Tengo mayor apreciación por el valor de mi } \\
\text { propia vida }\end{array}$ & 130 & 4 & 3,20 & 1,54 &, 63 \\
\hline 3. & He desarrollado muevos intereses & 126 & 3 & 2,43 & 1,57 &, 66 \\
\hline 4. & Me siento más autosuficiente & 129 & 2 & 1,88 & 1,63 &, 54 \\
\hline 5. & $\begin{array}{l}\text { Tengo mejor entendimiento de las cosas } \\
\text { espirituales }\end{array}$ & 128 & 2 & 2,02 & 1,79 &, 65 \\
\hline 6. & $\begin{array}{l}\text { Puedo ver más claramente que puedo contar } \\
\text { con otras personas en tiempos dificiles }\end{array}$ & 129 & 3 & 3,04 & 1,71 &, 66 \\
\hline 7. & Me abri un nuevo camino en mi vida & 129 & 2 & 1,94 & 1,72 &, 68 \\
\hline 8. & $\begin{array}{l}\text { Me siento más cercano/a, allegado/a, a otras } \\
\text { personas }\end{array}$ & 129 & 3 & 2,61 & 1,72 &, 80 \\
\hline 9. & $\begin{array}{l}\text { Puedo expresar mis emociones con más } \\
\text { facilidad }\end{array}$ & 130 & 3 & 2,32 & 1,57 &, 80 \\
\hline 10. & Sé que puedo enfrentar dificultades & 129 & 3 & 2,71 & 1,76 &, 78 \\
\hline 11. & Estoy capacitado/a para mejorar mi vida & 126 & 3 & 2,74 & 1,73 &, 79 \\
\hline 12. & $\begin{array}{l}\text { Me siento mejor capacitado/a para aceptar las } \\
\text { cosas como vengan }\end{array}$ & 129 & 3 & 2,69 & 1,68 &, 81 \\
\hline 13. & Siento mayor apreciación por cada día de vida & 127 & 4 & 3,22 & 1,69 &, 81 \\
\hline 14. & $\begin{array}{l}\text { Se me han presentado nuevas oportunidades } \\
\text { que no se me hubiesen presentado de otra } \\
\text { forma }\end{array}$ & 130 & 1 & 1,58 & 1,62 &, 62 \\
\hline 15. & Siento mayor compasión por los demás & 128 & 2 & 2,24 & 1,70 &, 60 \\
\hline 16. & Me esfuerzo más en mis relaciones personales & 129 & 3 & 2,49 & 1,68 &, 76 \\
\hline 17. & $\begin{array}{l}\text { Tengo mayor probabilidad de intentar cambiar } \\
\text { las cosas que necesitan cambios }\end{array}$ & 127 & 3 & 2,22 & 1,58 &, 68 \\
\hline 18. & Tengo más fe en mi religión & 129 & 1 & 1,48 & 1,71 &, 53 \\
\hline 19. & Descubrí que soy más fuerte de lo que pensaba & 130 & 3 & 2,88 & 1,84 &, 75 \\
\hline 20. & Aprendí que la gente es maravillosa & 129 & 3 & 2,47 & 1,69 &, 75 \\
\hline 21. & $\begin{array}{l}\text { Se me hace más fácil aceptar que necesito de } \\
\text { los demás }\end{array}$ & 129 & 3 & 2,73 & 1,68 &, 73 \\
\hline & & & & & \\
\hline
\end{tabular}

del modelo revisado muestran valores aceptables, una distribución $X^{2}$ $=57,53$ con 43 grados de libertad y con un valor $P>0,068$, estadisticamente no significativa. Los indicadores del ajuste global del modelo sugieren que el modelo es satisfactorio. La medida del $\mathrm{GFI}=0,926$ y $\mathrm{AGFI}=0,866$, en general el valor de estos Indices oscila entre 0 y 1 , cuando su valor se encuentra próximo a 1 el modelo se 
Tabla 3. Análisis factorial exploratorio de los factores del PTGI en pacientes oncológicos. Desestimadas cargas factoriales menores 0,25 .

Señaladas en negrita las saturaciones significativas en cada factor

\begin{tabular}{|c|c|c|c|c|c|}
\hline \multirow[t]{2}{*}{ Ítem } & \multirow{2}{*}{$\begin{array}{c}\text { Factor original del } \\
\text { PGTI }^{\star}\end{array}$} & \multicolumn{4}{|c|}{ Cargas factoriales } \\
\hline & & $\bar{I}$ & II & III & IV \\
\hline \multicolumn{6}{|c|}{ Factor I: $(54,47 \%$ varianza) } \\
\hline PGTI-12 & PS &, 825 & & 330 & \\
\hline PGTI-10 & PS &, 823 & & 301 & \\
\hline PGTI-11 & $\mathrm{NP}$ &, 792 & & &, 376 \\
\hline PGTI-20 & RO & ,699 & ,496 & & \\
\hline PGTI-19 & PS & ,676 & &, 334 & ,251 \\
\hline PGTI-21 & $\overline{\text { RO }}$ &, 597 & ,460 & & \\
\hline PGTI-17 & NP &, 552 & ,435 & & \\
\hline PTGI-9 & $\overline{\mathrm{RO}}$ &, $\mathbf{5 8 0}$ & &, 568 &, 281 \\
\hline \multicolumn{6}{|c|}{ Factor II: $(6,40 \%$ varianza $)$} \\
\hline PGTI-18 & $\mathrm{SC}$ & & ,828 & & \\
\hline PGTI-5 & $\mathrm{SC}$ & & ,674 & 296 &, 297 \\
\hline PGTI-15 & RO & &, 660 &, 297 & \\
\hline PTGI-14 & $\overline{N P}$ & ,349 &, $\mathbf{5 3 5}$ & ,399 & \\
\hline PGTI-16 & RO &, 449 &, 533 & ,338 &, 252 \\
\hline PGTI-7 & $\mathrm{NP}$ & 283 &, 457 & ,338 &, 412 \\
\hline \multicolumn{6}{|c|}{ Factor III: $(5,88 \%$ varianza) } \\
\hline PGTI-4 & PS & & &, 769 & \\
\hline PTGI-8 & $\overline{\mathrm{RO}}$ &, 479 &, 391 &, 587 & \\
\hline PTGI-6 & RO &, 462 & &, 571 & \\
\hline PTGI-3 & NP & & 253 & 564 &, 554 \\
\hline \multicolumn{6}{|c|}{ Factor IV: $(4,85 \%$ varianza $)$} \\
\hline PTGI-2 & $\mathrm{AL}$ & & &, 317 &, 854 \\
\hline PTGI-1 & $\overline{\mathrm{AL}}$ & 306 & & &, $\mathbf{8 1 0}$ \\
\hline PGTI-13 & $\mathrm{AL}$ &, 513 &, 282 & ,276 &, 615 \\
\hline
\end{tabular}

*AL: apreciación por la vida, NP: nuevas posibilidades, PS: fuerza personal, RO: relación con otros, SC: crecimiento espiritual. 
ajusta correctamente a los datos. En cambio son preferibles valores bajos al calcular el error de aproximación cuadrático medio (RMSEA), con oscilaciones entre 0,05 y 0,08 . Los resultados con esta medida también indica un ajuste aceptable del modelo, RMSEA $=0,054$. En la figura 1 presentamos el modelo monofactorial asumido tras el análisis de ecuaciones estructurales, la variable beneficios percibidos es una buena medida de su factor, puesto que los cuadrados de los coeficientes de correlación múltiple son mayores que 0,5 excepto en el caso del item $6(0,44)$ e item $17(0,46)$ que se sitúan relativamente cerca. La relación causal entre el factor beneficios percibidos y los ítems son adecuados, con unas correlaciones que oscilan entre 0,66 del item 6 a 0,90 en el ftem 12. La existencia de correlaciones entre los errores de medición nos advierten de la posible presencia de variables omitidas causantes de una variación común entre las variables superficiales cuyos residuos covarian.

La nueva estructura factorial del cuestionario está formada por 12 items que agrupan las dimensiones propuestas por los autores del cuestionario de fuerza personal, items 12, 10 y 19 (excepto ítem 4 «me siento más autosuficiente") y la dimensión relación con los demás, ítems $20,21,16,8,6$ y 9 (excepto ítem 15 «siento mayor compasión por los demás»), además se incluye el ítem 13 de la dimensión apreciación por la vida (usiento mayor apreciación por cada día de vida") y los ítems 11 y 17 de la dimensión nuevas posibilidades, referidos a la percepción de un cambio vital («estoy más capacitado/a para mejorar mi vida; «tengo mayor probabilidad de intentar cambiar las cosas que necesitan cambios»). El análisis de fiabilidad de la escala considerando la estructura monofactorial muestra un indice alfa de 0,95.

\section{Comparación de medias}

De la comparación de medias entre el género con la puntuación total del PTGI, se asume la igualdad de varianzas entre los dos grupos $(F=0,01 ; p \leq 0,91)$, no aparecen diferencias estadísticamente significativas entre hombres y mujeres $(T=1,18 ; p \leq 0,23)$, véase tabla 4. Respecto a la severidad del diagnóstico, graduado según estadio de la enfermedad, no aparecen diferencias significativas entre estadios con la puntuación total del PTGI $(F=0,14 ; p \leq 0,93)$, manteniendo 
Figura 1. Modelo estructural del PTGI, con parámetros estandarizados

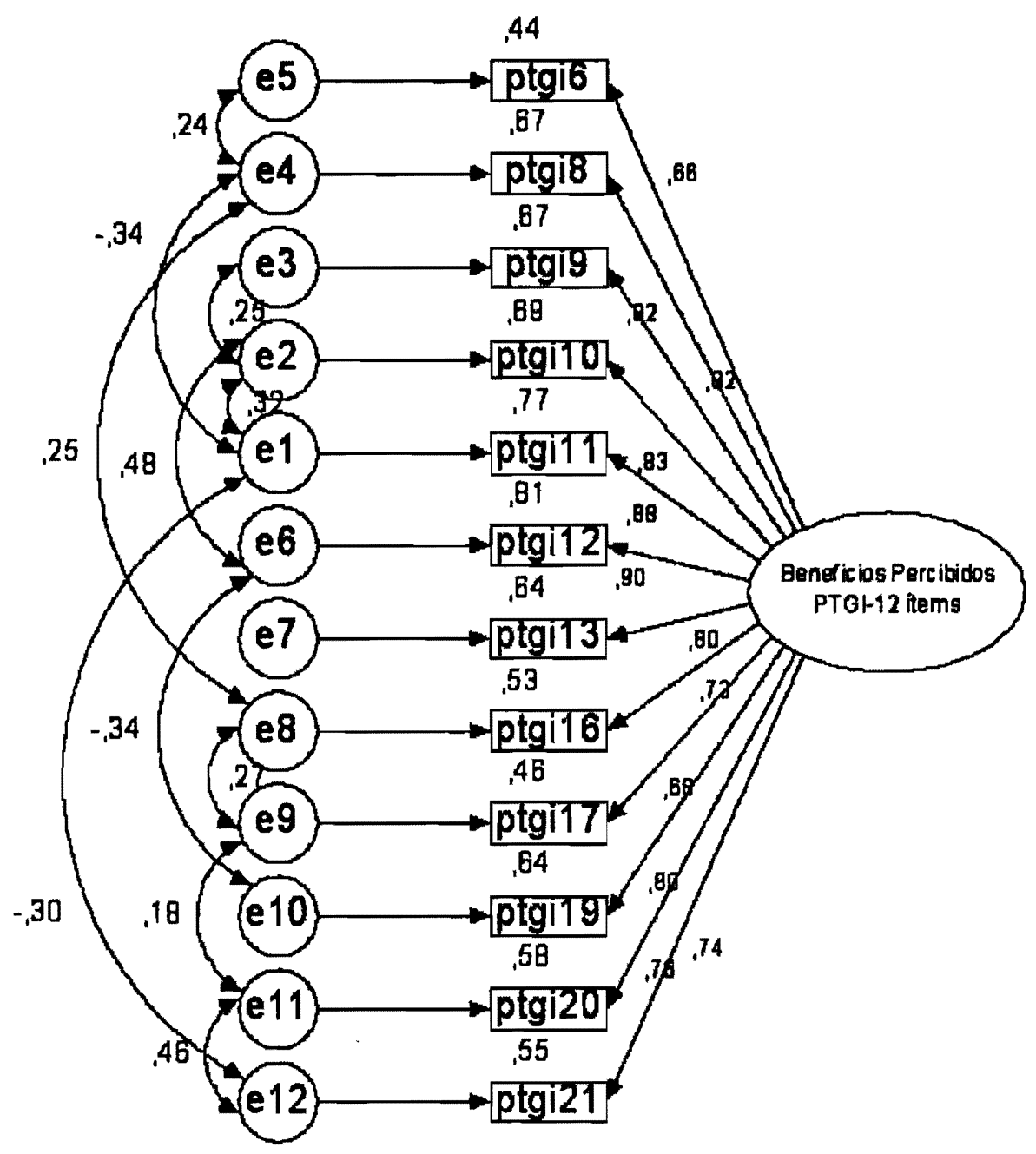

inicialmente la homogeneidad de vananzas entre los grupos $(F=1,92$; $p \leq 0,12)$. Del tratamiento médico recibido, contrastada la igualdad de varianzas entre grupos $(F=0,27 ; p \leq 0,60)$, el grupo que realizó quimioterapia tiende a mostrar más puntuación en el PTGI que el grupo que no realizó la quimioterapia, aunque las diferencias no llegan a ser significativas $(T=1,67 ; p \leq 0,09)$. Asumiendo la homogeneidad de 
varianzas $(F=1,53 ; p \leq 0,21)$, el no haberse sometido a tratamiento de radioterapia conlleva más crecimiento postraumático que haberse tratado con radioterapia aunque tampoco las diferencias son significativas $(T=-1,17 ; p \leq 0,24)$, véase tabla 4 .

Tabla 4. Puntuación media y desviación típica (Sx) en cada variable.

Comparaciones de medias con la prueba Tentre el PGTI, sexo y tratamiento médico actual (quimioterapia y radioterapia). Comparación de medias con el factor $F$ entre el PTGl y estadio clínico de la enfermedad (local, loco-regional, metastático, tumor no sólido)

\begin{tabular}{|lc|}
\hline & $\begin{array}{c}\text { Total-PGTI } \\
\text { Media(Sx) }\end{array}$ \\
\hline Sexo & \\
Varón & $33,71(16,59)$ \\
Mujer & $30,28(16,04)$ \\
Prueba $T$ & 1,18 \\
\hline Estadio & \\
Local & $32,68(14,60)$ \\
Loco-regional & $30,48(18,67)$ \\
Metastático & $32,58(16,74)$ \\
Tumor No-sólido & $31,76(16,30)$ \\
Factor $F$ & 0,146 \\
\hline Tratamiento médico & \\
Quimioterapia & \\
SI & $32,77(16,27)$ \\
NO & $26,05(14,98)$ \\
Prueba T & 1,67 \\
Radioterapia & \\
SI & $26,92(13,61)$ \\
No & $32,33(16,46)$ \\
Prueba $T$ & $-1,17$ \\
\hline *0 & \\
\hline
\end{tabular}

\section{Correlaciones}

No aparecen relaciones significativas entre la edad y beneficios percibidos tras el acontecimiento traumático $(r=0,9 ; p>0,05)$. El tiempo transcurrido desde el diagnóstico tampoco parece influir en la cantidad de crecimiento postraumático que una persona puede experimentar 
$(r=0,14 ; p>0,05)$, véase tabla 5 . La puntuación media en malestar emocional del HADS total $=9,5(S x=6,1)$, en sintomatología de ansiedad HADS-A= 5,9 $(S x=3,7)$ y en sintomatologia depresiva HADS-D=3,6 $(S x=3,2)$. En ningún caso la puntuación media llega al punto de corte establecido para considerar la sintomatologia como probable caso clínico (Zigmond y Snaith, 1983). En el análisis correlacional el malestar emocional referido a sintomatologia ansiosa o depresiva evaluada con el HADS no aparece asociada significativamente con crecimiento postraumático.

Tabla 5. Correlaciones entre PGTI, tiempo desde el diagnóstico y edad. Correlaciones entre el PGTI y las dimensiones del HADS

\begin{tabular}{|lc|}
\hline & Total-PGTI \\
\hline Edad & $-0,09$ \\
\hline Tiempo del diagnóstico & $-0,14$ \\
\hline HADS & \\
HADS-Total & 0,023 \\
HADS-Ansiedad & 0,063 \\
HADS-Depresion & $-0,029$ \\
\hline
\end{tabular}

\section{DIscusıón}

La adaptación española del PTGI en pacientes oncológicos aunque muestra buenas propiedades psicométricas, no permite replicar la estructura factorial de cinco dimensiones propuestas por los autores del cuestionario (Tedeschi y Calhoum, 1996). En la adaptación del PTGI a población oncológica china, utilizando el análisis factorial confirmatorio se comprueba un modelo de dos factores, una dimensión interpersonal y otra intrapersonal, que explica la percepción de crecimiento postraumático (Ho y cols., 2004). En la muestra de este estudio, el cuestionario conforma una estructura monofactorial que permite contrastar la validez estructural del concepto y cuantificar los beneficios percibidos tras el diagnóstico o tratamiento de la enfermedad oncológica. En el modelo estructural extraído para el PTGI la existencia de residuos correlacionados entre variables observadas puede esconder la presencia de otros factores o dimensiones causantes de la variación, aunque según los resultados no sean significativos. 
EI PTGI nos permite evaluar el cambio en sentido positivo que experimenta la persona en la percepción de sí misma y de la relación con los demás por el afrontamiento de la enfermedad. Según el significado de la agrupación monofactorial de items podríamos decir que tras la enfermedad la persona confirma su fortaleza para superar dificultades por haber sobrevivido a lo peor, se siente más cercana y unida a los demás ante las dificultades, hay una voluntad para dejarse ayudar o utilizar el apoyo social que antes habla ignorado. El apoyo social mejora la respuesta cognitiva (pensamientos intrusivos y búsqueda de significado) ante la enfermedad oncológica, además es considerada una variable que influye en el bienestar emocional, o incluso, sobre el tiempo de supervivencia (Roberts y cols., 2006; Kornblith y cols., 2001; Frick y cols., 2005).

Por otra parte el no poder replicar la estructura factorial propuesta por los autores del cuestionario (Tedeschi y Calhoum, 1996), nos señala la falta de uniformidad en la definición del concepto como refieren otros estudios (Lechner y cols., 2003; Carver y cols., 2002; Helgeson y cols., 2006). En este sentido algunos autores para referirse a crecimiento postraumático prefieren utilizar el término beneficio percibido (traducción del inglés de benedit finding o perceived benefits; Helgeson ycols., 2006). Además hay que tener en cuenta que las características de la muestra y su encuadre socio-cultural determina la forma en que se manifiesta el crecimiento postraumático (Calhoun y Tedeschi, 2004).

Respecto a las variables sociodemográficas, no aparecen diferencias entre hombres y mujeres en crecimiento postraumático. La mayoría de estudios realizados sobre crecimiento postraumático en pacientes oncológicos están basados en mujeres con cáncer de mama (Cordova y cols., 2001; Bellizi y cols., 2006; Manne y cols., 2004; Weiss, 2004), de los pocos estudios con una muestra heterogénea en algunos no aparecen diferencias entre hombres y mujeres en crecimiento postraumático (Ho y cols., 2004; Lechner y cols., 2003) y en otros, son las mujeres las que perciben más beneficio tras el diagnóstico o tratamiento oncológico( Jaarsma y cols., 2006; Carboon y cols., 2005).

En este estudio, la edad no muestra correlaciones significativas con las dimensiones del PTGl, este resultado es semejante al obtenido en un estudio realizado en pacientes con cáncer de próstata (Thornton 
y cols., 2006) pero que difiere del mostrado en la literatura, es decir la edad correlaciona de forma negativa y significativa con crecimiento postraumático (Tedeschi y Calhoum, 1996; Jaarsma y cols., 2006; Bellizzi y cols., 2006; Manne y cols., 2004; Lechner y cols., 2003), las personas jóvenes perciben un mayor cambio positivo tras una experiencia traumática como asumir el diagnóstico o tratamiento de una enfermedad oncológica.

Durante el primer año tras el diagnóstico del cáncer la persona queda con un primer impacto emocional y absorbida por el tratamiento oncológico, necesita un tiempo para asumir y madurar el diagnóstico o tratamiento de la enfermedad (Cordova y cols., 2001; Calhoum y Tedeschi, 2006; Jaarsma y cols., 2006). En nuestro estudio no aparecen correlaciones significativas entre tiempo desde el diagnóstico y crecimiento postraumático. Algunos autores al no encontrar relación entre tiempo desde el diagnóstico y beneficios percibidos tras el acontecimiento traumático (Lechner y cols., 2003), se plantean estudiar cómo influye el grado de crecimiento postraumático durante el primer año de tratamiento con una respuesta adaptativa a largo plazo, es decir, los beneficios percibidos correlacionan con menos malestar emocional y mejor calidad de vida a los 5 y 8 años después de finalizar el tratamiento (Carver y cols., 2004).

La intensidad del acontecimiento traumático genera potencialmente más cambio personal en sentido positivo (Tedeschi y Calhoum, 1996). En este estudio la intensidad del acontecimiento traumático se valora con la gravedad de la enfermedad (estadio) y severidad del tratamiento médico recibido (quimioterapia o radioterapia). No aparecen diferencias significativas entre los diferentes estadios de la enfermedad y puntuaciones en el PTGI, aunque los pacientes en tratamiento con quimioterapia tienden a experimentar más crecimiento postraumático, esta diferencia no es significativa. De la relación entre beneficio percibido y severidad del acontecimiento traumático, algunos estudios tratan de establecer una relación curvilinea (Lechner y cols., 2006), pacientes con estadio Il de la enfermedad informan de más beneficio percibido que pacientes con estadio I o IV (Bellizi y cols., 2006; Lechner y cols., 2003; Lechner y cols., 2006).

En pacientes oncológicos se han encontrado correlaciones negativas entre malestar emocional y crecimiento postraumático (Ho y cols., 2004). 
En este estudio las puntuaciones medias en el HADS no llegan a los puntos de corte establecidos para considerar la sintomatología como probable caso clinico, quizá esto pueda explicar que la sintomatología de ansiedad y depresión o el malestar emocional, evaluado con el HADS, no aparece relacionado con la dimensión del PGTI, estos resultados son semejantes a otros estudios y no resultan aclaratorios (Ho y cols., 2004; Jaarsma y cols., 2006; Helgeson y cols., 2006).

Este estudio presenta algunas limitaciones que cabe mencionar, primero las referidas al tamaño muestral, aunque están dentro de niveles estadisticamente aceptables se podria considerar el incrementar su tamaño para profundizar en un estudio clínico de las variables sociodemográficas y médicas relacionadas con el cuestionario. Al considerar el estadio de la enfermedad y hallar su relación con crecimiento postraumático, habria que especificar la gravedad del diagnóstico con otros indicadores más reales, como agresividad del tumor, afectación ganglionar o tipo de metástasis, y no sólo por la extensión del tumor (local, loco-regional o metastático). Finalmente, a nivel metodológico en el estudio de variables como tiempo desde el diagnóstico, o severidad del acontecimiento traumático y crecimiento postraumático parece muy simplificado el utlizar modelos lineales de regresión para explicar una relación entre las variables que se muestra más compleja al analizarlo con cierta profundidad.

Con este trabajo damos unos primeros pasos en el estudio del sesgo o evaluación positiva que tiene el paciente oncológico ante el diagnóstico o tratamiento de la enfermedad, otros aspectos quedan todavía a considerar.

\section{REFERENCIAS BIBLIOGRÁFICAS}

American Psychiatric Association. (1995). Manual diagnóstico y estadistico de los trastornos mentales. $4^{\text {a }}$ ed. Barcelona:Masson. Bellizzi KM, Blank TO. (2006). Predicting posttraumatic growth in breast cancer survivors. Health Psychology, 25 (1): 47-56.

Calhoun LG, Tedeschi RG (2004). The foundations of posttraumatic growth: new considerations. Psychology Inquiry, 15(1): 93-102.

Calhoum LG, Tedeschi RG.(2006). The Foundations of Posttraumatic Growth: an expanded framework. En: Calhoum LG, Tedeschi RG, 
editors. Mahwah, NJ: Lawrence Erlbaum Associate Publishers; p.3-23.

Carboon I, Anderson VA, Pollard A, Szer J, Seymour JF. (2005).

Posttraumatic Growth following a cancer diagnosis: do world assumptions contribute? Traumatology, 11(4): 269-283.

Carver CS, Antoni MH. (2004). Finding benefit in breast cancer during the year after diagnosis predicts better adjustment 5 to 8 years after diagnosis. Health psychology; 23(6): 595-598.

Cronbach LJ. (1951). Coefficient alpha and the internal structure of test. Psychometrika; 16: 297-334.

Cordova MJ, Cunningham LL, Carison CR, Andrykowski MA. (2001).

Posttraumatic growth following breast cancer: a controlled comparasion study. Health Psychology, 20 (3): 176-185.

Frick E. Motzke C, Fischer N, Busch N, Bumeder I. (2005). Is perceived social support a predictor of survival for patients undergoing autologous peripheral blood stem cell transplantation? Psychooncology, 14: 759-770.

Gorsuch RL. (1983). Factor Analysis. 2ed. Hillsdale, New Jersey: Lawrence Erlbaum Associates.

Helgeson VS, Reynolds KA, Tomich PL. (2006). A meta-analytic review of benefit finding and growth. Journal of consulting and clinical psychology; 74(5): 797-816.

Ho SMY, Chan CLW, Ho RTH. (2004). Posttraumatic growth in chinese cancer survivors. Psycho-oncology; 13: 377-389.

Jaarsma TA, Pool G, Sanderman R, Ranchor AV. (2006). Psychometric properties of the dutch version of the posttraumatic growth inventory among cancer patients. Psycho-oncology; 15(10): 911-920.

Karnofsky DA, Abelman WH, Craver LF et al. (1948). The use of nitrogen mustards in the palliative treatment of carcinoma. Cancer; 1: 634656.

Kornblith AB, Herndon JE, Zuckerman E, Viscoli CM, Horwitz RI, Cooper MR, Harris L, Tkaczuk KH, Perry MC, Budman D, Norton L, Holland JC. (2001). Social support as a buffer to the psychological impact of stressful life events in women with breast cancer. Cancer; 91 (2): 443-454.

Lechner SC, Zakowski SG, Antoni MH, Greenhawt M, Block K, Block P. (2003). Do sociodemographic and disease-related variables 
influence benefit-finding in cancer patients?. Psycho-oncology, 12: 491-499.

Lechner SC, Carver CS, Antoni MH, Weaver KE, Phillips KM. (2006). Curvilinear associations between benefit finding and psychosocial adjustment to breast cancer. Joumal of consulting and clinical psychology, 74(5): 828-840.

López-Roig S, Terol MC, Neipp MC, Massuti B, Rodríguez-Marín J, Leyda JL, Martín-Aragón M, Sitges E. Ansiedad y Depresión. (2000). Validación de la escala HAD en pacientes oncológicos. Revista de Psicología de la Salud; 12 (2):127-155.

Manne S, Ostrff J, Winkel G, Goldstein L, Fox K, Grana G. (2004). Posttraumatic growth after breast cancer. patient, partner and couple perspectives. Psychosomatic Medicine; 66: 442-454.

Pfeiffer E. (1975). A short portable mental status questionnaire for the assesment of organic brain deficit in elderly patients. JAm Geriatrics Soc; 23: 433-41.

Ramos MM, Catena A, Trujillo HM. (2004). Modelos de ecuaciones estructurales. En: Manual de métodos y técnicas de investigación en ciencias del comportamiento. Madrid: Biblioteca nueva.

Roberts KJ, Lepore SJ, Helgeson V. (2006). Social-cognitive correlates of adjustment to prostate cancer. Psycho-Oncology; 15: 183-192. Tedeschi RG, Calhoum LG. (1995). Trauma and transformation: Growing in the aftermath of suffering. Thousand Oaks, CA: Sage Publications.

Tedeschi RG, Calhoum LG. (1996). The posttraumatic Growth Inventory: Measuning the Positive Legacy of Trauma. Journal of Traumatic Stress; 9 (3): 455-471.

Thornton AA, Martin AP. (2006). Posttraumatic growth in prostate cancer

survivors and their partners. Psycho-Oncology; 15:285-296.

Weiss T, (2004). Correlates of posttraumatic growth in married breast cancer survivors. Journal of Social and Clinical Psychology ; 23(5): 733-746.

Weiss T, Berger R. (2005). Reliability and Validity of a Spanish version of the Posttraumatic Growtn Inventory. Research on social work practice; 20(10): 1-9.

Zigmond AS, Snaith RP.(1983). The Hospital Anxiety and Depresión Scale. Acta Psychiatr. Scand; 67: 361-370. 